

\title{
Partial observer normal form for nonlinear system
}

Ramdane Tami, Gang Zheng, Driss Boutat, Didier Aubry, Haoping Wang

\section{To cite this version:}

Ramdane Tami, Gang Zheng, Driss Boutat, Didier Aubry, Haoping Wang. Partial observer normal form for nonlinear system. Automatica, 2016, 64, pp.54-62. 10.1016/j.automatica.2015.10.041 . hal01239583

\section{HAL Id: hal-01239583 \\ https://inria.hal.science/hal-01239583}

Submitted on 8 Dec 2015

HAL is a multi-disciplinary open access archive for the deposit and dissemination of scientific research documents, whether they are published or not. The documents may come from teaching and research institutions in France or abroad, or from public or private research centers.
L'archive ouverte pluridisciplinaire HAL, est destinée au dépôt et à la diffusion de documents scientifiques de niveau recherche, publiés ou non, émanant des établissements d'enseignement et de recherche français ou étrangers, des laboratoires publics ou privés. 


\title{
Partial observer normal form for nonlinear system ${ }^{\text {th }}$
}

\author{
Ramdane Tami ${ }^{\mathrm{a}}$, Gang Zheng ${ }^{\mathrm{b}, \mathrm{c}}$, Driss Boutat $^{\mathrm{a}}{ }^{\text {, Didier Aubry }}{ }^{\mathrm{d}}$, Haoping Wang ${ }^{\mathrm{e}, 1}$ \\ ${ }^{a}$ INSA Centre Val de Loire, univ Orléans, institut PRISME UPRES 4229, 88 BLD Lahitolle, 18020 Bourges Cedex, France \\ ${ }^{b}$ Non-A, INRIA - Lille Nord Europe, 40 avenue Halley, Villeneuve d'Ascq 59650, France. \\ ${ }^{c}$ CRIStAL, CNRS UMR 9189, Ecole Centrale de Lille, BP 48, 59651 Villeneuve dAscq, France. \\ ${ }^{d}$ IUT de Orleans, univ Orleans, institut PRISME UPRES 4229, 63 av. de Lattre de Tassigny, 18020 Bourges, France \\ ${ }^{e}$ School of Automation. Nanjing University of Science and Technology, 210094 Nanjing, China
}

\begin{abstract}
In this paper, we investigate the estimation problem for a class of partially observable nonlinear systems. For the proposed Partial Observer Normal Form (PONF), necessary and sufficient conditions are deduced to guarantee the existence of a change of coordinates which can transform the studied system into the proposed PONF. Examples are provided to illustrate the effectiveness of the proposed results.
\end{abstract}

Keywords: Observer normal form; Partial state observer; Nonlinear systems

\section{Introduction}

Observability and observer design problem for nonlinear systems have been widely studied during last four decades, and many different methods have been proposed to treat different classes of nonlinear systems, such as adaptive observer, high-gain observer, sliding mode observer and so on Besançon (2007). In this work, we are interested in the approach based on geometrical transformations to bring the original system into a simple observer normal form. The advantage of this method is that, by well choosing the desired simple observer normal form, we can reuse the existing observers proposed in the literature to estimate the state of the transformed observer normal form, and then obtain the state estimation for the original system by inverting the deduced diffeomorphism. The literature about this technique is vast. Since the pioneer works of (Bestle and Zeitz (1983); Krener and Isidori (1983)) for single output systems and (Krener and Respondek (1985); Xia and Gao (1989)) for the case of MIMO systems, many other results (see Keller (1987); Marino and Tomei (1996); Lynch and Bortoff (2001); Phelps (1991); Boutat et al. (2009)) were published by following the same idea. However, the solvability of the problem requires the restrictive commutative Lie bracket condition for the deduced vector fields. In order to relax this restriction,

\footnotetext{
This paper was supported in part by Région Centre France, by Ministry of Higher Education and Research Nord-Pas de Calais Regional Council, by FEDER through the Contrat de Projets Etat Region (CPER), by ARCIR Project ESTIREZ Nord-Pas de Calais Regional Council, by Chinses NSF(61304077), and by International Science Technology Cooperation Program of China (2015DFA01710).

Email addresses: ramdane.tami@insa-cvl .fr (Ramdane Tami), gang .zheng@inria.fr (Gang Zheng), driss . boutat@insa-cvl.fr (Driss Boutat), Didier.Aubry@univ-orleans.fr (Didier Aubry), hp.wang@njust.edu.cn (Haoping Wang)

${ }^{1}$ Corresponding author.
}

we can reconstruct a new family of vector fields which can satisfy the commutative Lie bracket condition. Inspired by this solution, various extensions are developed for output depending nonlinear observer normal form (Guay (2002); Respondek et al. (2004); Zheng et al. (2005, 2009, 2007); Boutat et al. (2006)) and for the extended nonlinear observer normal form (Jouan (2003); Noh et al. (2004); Back et al. (2006); Boutat and Busawon (2011); Tami et al. (2013); Boutat (2015)). The related applications can be found as well for the synchronization of nonlinear systems Zheng and Boutat (2011), for Dengue epidemic model Tami et al. (2014b), for PM synchronous motor Tami et al. (2014a), and so on.

Most of the references cited above are devoted to designing a full-order observer, under the assumption that the whole state of the studied system is observable. Few works have been dedicated to the partial observability which however makes sense in practice when only a part of states are observable or are necessary for the controller design. Among the works on this issue, we can cite the work of Kang et al. (2009) where the authors gave a general definition of observability covering the partial one. Reduced-order observer and LMI technique are proposed in Trinh et al. (2006) to estimate the part of observable states. In Robenack and Lynch (2006), the authors proposed a general partial nonlinear observer canonical form and used the geometrical method transforming a nonlinear dynamical system into this observer form. However, the nonlinear term in this canonical form contains as well the unobservable states. Thus, to design an observer for the proposed canonical form, one needs the Lipschitz condition to guarantee the estimation convergence. Jo and Seo (2002) provided necessary and sufficient geometrical conditions which guarantee the existence of a change of coordinates transforming a nonlinear dynamical system into a special partial observer normal form. This normal form is divided into two dynamical subsystems: the first one contains only a part 
of states which is of Brunovsky canonical form modulo output injection; the second one is nonlinear containing only the unobservable states and the output. This result can be seen as a direct application of the result of Krener and Isidori (1983) to the partial family of vector fields, therefore it suffers from the same restriction on the commutative Lie bracket condition.

In order to relax the restriction of the result presented in Jo and Seo (2002), this paper proposes a more general PONF for a class of partially observable nonlinear systems. This new form is a generalization of the normal form studied in Jo and Seo (2002). The proposed PONF is divided as well into two subsystems, and we relax the form proposed in Jo and Seo (2002) by involving all states in the second subsystem. To deal with this generalization, we use the notion of commutativity of Lie bracket modulo a distribution. Moreover, our results allow as well to apply additionally a diffeomorphism on the output space. Therefore, the necessary and sufficient geometrical conditions established in this paper are more general and quite different from those stated in Jo and Seo (2002) because of the introduction of commutativity of Lie bracket modulo a distribution.

The paper is organized as follows. Section 2 is devoted to the technical background and problem statement. In Section 3, some preliminary results are given and then necessary and sufficient conditions allowing the construction of PONF are stated. Section 4 generalizes the result in the previous section by applying a change of coordinates on the output. In the end, an example of Susceptible, Infected and Removed (SIR) epidemic model is presented to highlight the proposed results.

\section{Notation and problem statement}

We consider the following nonlinear dynamical system with single output:

$$
\begin{aligned}
\dot{x} & =f(x)+g(x) u=f(x)+\sum_{k=1}^{m} g_{k}(x) u_{k} \\
y & =h(x)
\end{aligned}
$$

where $x \in \mathbb{R}^{n}, u \in \mathbb{R}^{m}, y \in \mathbb{R}$, and the functions $f: \mathbb{R}^{n} \rightarrow \mathbb{R}^{n}$, $g=\left[g_{1}, \cdots, g_{m}\right]$ with $g_{i}: \mathbb{R}^{n} \rightarrow \mathbb{R}^{n}$ for $1 \leq i \leq m, h: \mathbb{R}^{n} \rightarrow \mathbb{R}$ are supposed to be sufficiently smooth. In this paper, it is assumed that $f(0)=0$ and $h(0)=0$.

Let $\mathscr{X} \subset \mathbb{R}^{n}$ be a neighborhood of 0 , for system (1)-(2), if the pair $(h(x), f(x))$ locally satisfies the observability rank condition on $\mathscr{X}$, i.e. $\operatorname{rank}\left\{d h, d L_{f} h, \cdots, d L_{f}^{n} h\right\}(x)=n$ for $x \in \mathscr{X}$, then the following 1-forms:

$$
\theta_{1}=d h \text { and } \theta_{i}=d L_{f}^{i-1} h, \text { for } 2 \leq i \leq n
$$

are independent on $\mathscr{X}$, where $L_{f}^{k} h$ denotes the $k^{t h}$ Lie derivative of $h$ along $f$. Therefore, there exists a family of vector fields $\bar{\tau}=\left[\bar{\tau}_{1}, \cdots, \bar{\tau}_{n}\right]$ proposed in Krener and Isidori (1983), where the first vector field $\bar{\tau}_{1}$ is the solution of the following algebraic equations:

$$
\left\{\begin{array}{l}
\theta_{i}\left(\bar{\tau}_{1}\right)=0 \text { for } 1 \leq i \leq n-1 \\
\theta_{n}\left(\bar{\tau}_{1}\right)=1
\end{array}\right.
$$

and the other vector fields are obtained by induction as $\bar{\tau}_{i}=$ $-a d_{f} \bar{\tau}_{i-1}=\left[\bar{\tau}_{i-1}, f\right]$ for $2 \leq i \leq n$, where [,] denotes the Lie bracket. According to Krener and Isidori (1983), if

$$
\begin{aligned}
& {\left[\bar{\tau}_{i}, \bar{\tau}_{j}\right]=0 \text { for } 1 \leq i, j \leq n} \\
& {\left[\bar{\tau}_{i}, g_{k}\right]=0 \text { for } 1 \leq i \leq n-1 \text { and } 1 \leq k \leq m}
\end{aligned}
$$

then system (1)-(2) can be locally transformed, by means of a local diffeomorphism $\xi=\phi(x)$, into the following nonlinear observer normal form:

$$
\left\{\begin{array}{l}
\dot{\xi}=A \xi+B(y)+\sum_{k=1}^{m} \alpha_{k}(y) u_{k} \\
y=C \xi
\end{array}\right.
$$

where $A \in \mathbb{R}^{n \times n}$ is the Brunovsky matrix and $C=[0, \cdots, 0,1] \in$ $\mathbb{R}^{1 \times n}$.

Obviously, for the nonlinear dynamical system (1)-(2), if $\operatorname{rank}\left\{d h, d L_{f} h, \cdots, d L_{f}^{n} h\right\}(x)=r<n$ for $x \in \mathscr{X}$, which implies that only a part of states of the studied system are observable, the proposed method by Krener and Isidori (1983) could not be applied.

To treat this partially observable situation, this paper proposes the following Partial Observer Normal Form (PONF):

$$
\left\{\begin{array}{l}
\dot{\xi}=A \xi+\beta(y)+\sum_{k=1}^{m} \alpha_{k}^{1}(y) u_{k} \\
\dot{\zeta}=\eta(\xi, \zeta)+\sum_{k=1}^{m} \alpha_{k}^{2}(\xi, \zeta) u_{k} \\
y=C \xi
\end{array}\right.
$$

where $\xi \in \mathbb{R}^{r}, \zeta \in \mathbb{R}^{n-r}, y \in \mathbb{R}, \beta: \mathbb{R} \rightarrow \mathbb{R}^{r}, \eta: \mathbb{R}^{r} \times \mathbb{R}^{n-r} \rightarrow$ $\mathbb{R}^{n-r}, \alpha_{k}^{1}: \mathbb{R} \rightarrow \mathbb{R}^{r}, \alpha_{k}^{2}: \mathbb{R}^{r} \times \mathbb{R}^{n-r} \rightarrow \mathbb{R}^{n-r}, C=(0, \cdots, 0,1) \in$ $\mathbb{R}^{1 \times r}$ and $A$ is the $r \times r$ Brunovsky matrix:

$$
A=\left(\begin{array}{ccccc}
0 & 0 & \cdots & 0 & 0 \\
1 & 0 & \ddots & \vdots & \vdots \\
0 & 1 & \cdots & 0 & 0 \\
0 & \cdots & \cdots & 0 & 0 \\
0 & \cdots & \cdots & 1 & 0
\end{array}\right) \in \mathbb{R}^{r \times r}
$$

For the proposed form (7), one can easily design a reducedorder observer to estimate the part of observable state $\xi$, by choosing the gain $K$ such that $(A-K C)$ is Hurwitz. Therefore, the rest of this paper focuses only on how to deduce a diffeomorphism which transforms the nonlinear system (1)-(2) into the proposed PONF (7).

Before dealing with this problem, let use highlight the fundamental difference between the proposed PONF with respect to the canonical form proposed in Jo and Seo (2002).

Remark 1. The normal form described in (7) is a generalization of the normal form obtained in Jo and Seo (2002). Indeed, in Jo and Seo (2002) the unobservable dynamics (the second dynamics $\zeta$ ) does not contain the observable state $\xi$. Let us consider the following modified example from Jo and Seo (2002) to highlight this difference:

$$
\left\{\begin{array}{l}
\dot{\xi}_{1}=u \\
\dot{\xi}_{2}=\xi_{1}+y^{2} \\
\dot{\zeta}=-\zeta+\mu \xi_{1}^{2}+y \\
y=\xi_{2}
\end{array}\right.
$$


If $\mu=0$, then this example is the same one studied in Jo and Seo (2002) and is of the normal form proposed in Jo and Seo (2002). However, if $\mu \neq 0$, then it is of the proposed normal form (7). Moreover, the conditions stated in Jo and Seo (2002) are not fulfilled for this example when $\mu \neq 0$. This fact will be discussed in Example 1.

\section{Geometrical conditions}

In this section, we will deduce necessary and sufficient conditions which guarantee the existence of a diffeomorphism to transform the studied partial observer nonlinear system into the proposed PONF. For this, considering the studied system (1)-(2), it is assumed in this paper that $\operatorname{rank}\left\{d h, d L_{f} h, \cdots, d L_{f}^{n} h\right\}(x)=r<n$ for $x \in \mathscr{X} \subset \mathbb{R}^{n}$, where $\mathscr{X}$ is a neighborhood of 0 .

Denote the observability 1 -forms for $1 \leq i \leq r$ by $\theta_{i}=$ $d L_{f}^{i-1} h$ and note $\Delta=\operatorname{span}\left\{\theta_{1}, \theta_{2}, \cdots, \theta_{r}\right\}$ as the co-distribution spanned by the observability 1 -forms. Specifically, thanks to observability rank condition, it is clear that $d L_{f}^{i} h \in \Delta$ for $i \geq r+1$. Then one can define the kernel (or the annihilator) of the co-distribution $\Delta$ as follows:

$$
\Delta^{\perp}=\operatorname{ker} \Delta=\left\{X: \theta_{k}(X)=0, \text { for } 1 \leq k \leq r\right\}
$$

for which we have the following properties which will be used in the sequel.

Lemma 1. For the distribution $\Delta^{\perp}$ defined in (9), the following properties are satisfied:

P1: $\Delta^{\perp}$ is involutive, i.e. for any two vector fields $H_{1} \in \Delta^{\perp}$ and $H_{2} \in \Delta^{\perp}$, we have $\left[H_{1}, H_{2}\right] \in \Delta^{\perp}$;

P2: there exist $(n-r)$ vector fields $\left\{\tau_{r+1}, \cdots, \tau_{n}\right\}$ that span $\Delta^{\perp}$ such that $\left[\tau_{i}, \tau_{j}\right]=0$ for $r+1 \leq i, j \leq n$, i.e. $\left\{\tau_{r+1}, \cdots, \tau_{n}\right\}$ is the commutative basis of $\Delta^{\perp}$;

P3: $\Delta^{\perp}$ is $f$-invariant, i.e. for any vector field $H \in \Delta^{\perp}$, we have $[f, H] \in \Delta^{\perp}$.

Proof. See Appendix A.1.

Now, let $\bar{\tau}_{1}$ be one of the vector field solutions of the following under-determined algebraic equations:

$$
\left\{\begin{array}{l}
\theta_{k}\left(\bar{\tau}_{1}\right)=0 \text { for } 1 \leq k \leq r-1 \\
\theta_{r}\left(\bar{\tau}_{1}\right)=1
\end{array}\right.
$$

and it is obvious that this solution is not unique, since (10) contains only $r$ algebraic equations. Therefore, for any $H \in \Delta^{\perp}$, $\bar{\tau}_{1}+H$ is also a solution of (10). By following the method proposed in Krener and Isidori (1983), we can construct, for any chosen $\bar{\tau}_{1}$ satisfying (10), the following vector fields:

$$
\bar{\tau}_{i}=\left[\bar{\tau}_{i-1}, f\right] \text { for } 2 \leq i \leq r
$$

Let $\tilde{\tau}_{1}$ be another solution of (10), which enables us, by following the same method, to construct another family of $(r-1)$ vector fields:

$$
\tilde{\tau}_{i}=\left[\tilde{\tau}_{i-1}, f\right] \text { for } 2 \leq i \leq r
$$

Then the following lemma highlights the relation between these two families of vector fields for the different chosen $\bar{\tau}_{1}$ and $\tilde{\tau}_{1}$.
Lemma 2. For any two different solutions $\bar{\tau}_{1}$ and $\tilde{\tau}_{1}$ of (10), there exist $H_{i} \in \Delta^{\perp}$ for $1 \leq i \leq r$ such that $\tilde{\tau}_{i}=\bar{\tau}_{i}+H_{i}$ where $\bar{\tau}_{i}$ and $\tilde{\tau}_{i}$ are defined in (11) and (12), respectively.

\section{Proof. See Appendix A.2.}

The above lemma reveals an important property: no matter how to choose the first vector field $\bar{\tau}_{1}$ satisfying (10), the family of vector fields $\bar{\tau}_{i}$ for $1 \leq i \leq r$ are defined modulo $\Delta^{\perp}$. Other important properties for this family of vector fields $\bar{\tau}_{i}$ are listed in the following lemma.

Lemma 3. For any $\bar{\tau}_{1}$ satisfying (10) and the associated $\bar{\tau}_{i}$ for $2 \leq i \leq r$ deduced from (11), the following properties are satisfied:

1) for any $H \in \Delta^{\perp}$, we have $\left[\bar{\tau}_{i}, H\right] \in \Delta^{\perp}$;

2) for any $H_{i} \in \Delta^{\perp}$ and $H_{j} \in \Delta^{\perp}$ with $1 \leq i, j \leq r$, we have

$$
\left[\bar{\tau}_{i}+H_{i}, \bar{\tau}_{j}+H_{j}\right]=\left[\bar{\tau}_{i}, \bar{\tau}_{j}\right] \text { modulo } \Delta^{\perp} ;
$$

3) for $1 \leq i \leq r$ and $r+1 \leq j \leq n$, we have

$$
\left[\bar{\tau}_{i}, \tau_{j}\right] \in \Delta^{\perp}
$$

where $\tau_{j}$ was defined in P2 of Lemma 1, which is the commutative basis of $\Delta^{\perp}$.

Proof. See Appendix A.3.

Lemma 4. For any $\bar{\tau}_{1}$ satisfying (10) and the associated $\bar{\tau}_{i}$ for $2 \leq i \leq r$ deduced from (11), there exists a family of vector fields $\tau_{i}=\bar{\tau}_{i}$ modulo $\Delta^{\perp}$ such that $\left[\tau_{i}, \tau_{j}\right]=0$ for $1 \leq i, j \leq r$, if and only if $\left[\bar{\tau}_{i}, \bar{\tau}_{j}\right] \in \Delta^{\perp}$.

Proof. See Appendix A.4.

Remark 2. Any family of vector fields $\bar{\tau}_{i}$ for $1 \leq i \leq r$ given by (10)-(11) together with the family of vector fields $\tau_{j}$ for $r+1 \leq$ $i \leq n$ defined in P2 of Lemma 1, are independent on $\mathscr{X}$. Thus they provide a basis of the tangent fiber bundle $T \mathscr{X}$ of $\mathscr{X}$.

The following example is for the purpose of highlighting the above results.

Example 1. Let us consider again system (8). A straightforward calculation shows that $\theta_{1}=d \xi_{2}$ and $\theta_{2}=d \xi_{1}+2 \xi_{2} d \xi_{2}$. Then we have $\Delta=\operatorname{span}\left\{\theta_{1}, \theta_{2}\right\}$, thus $\Delta^{\perp}=\operatorname{span}\left\{\frac{\partial}{\partial \zeta}\right\}$. According to (10), we obtain $\bar{\tau}_{1}=\frac{\partial}{\partial \xi_{1}}+q_{1}\left(\xi_{1}, \xi_{2}, \zeta\right) \frac{\partial}{\partial \zeta}$ where $q_{1}\left(\xi_{1}, \xi_{2}, \zeta\right)$ being any function of its arguments. Following (11), we have: $\bar{\tau}_{2}=\left[\bar{\tau}_{1}, f\right]=\frac{\partial}{\partial \xi_{2}}+q_{2} \frac{\partial}{\partial \zeta}$ with $q_{2}=2 \mu \xi_{1}-$ $q_{1}-\frac{\partial q_{1}}{\partial \xi_{2}}\left(\xi_{1}+\xi_{2}^{2}\right)-\frac{\partial q_{1}}{\partial \zeta}\left(-\zeta+\mu \xi_{1}^{2}+\xi_{2}\right)$. The Lie bracket of these two vector fields yields:

$$
\left[\bar{\tau}_{1}, \bar{\tau}_{2}\right]=\left[\frac{\partial q_{2}}{\partial \xi_{1}}+\frac{\partial q_{2}}{\partial \zeta} q_{1}-\frac{\partial q_{1}}{\partial \xi_{2}}-\frac{\partial q_{1}}{\partial \zeta} q_{2}\right] \frac{\partial}{\partial \zeta}
$$

which is not equal to 0 if $q_{1} \neq 0$ and $\mu \neq 0$. Therefore, the conditions stated in Jo and Seo (2002) are not fulfilled. However, we have $\left[\bar{\tau}_{1}, \bar{\tau}_{2}\right] \in \Delta^{\perp}$. Therefore, thanks to Lemma 4 we can found two other vector fields which are commutative.

It is easy to check that, by choosing $H_{1}=-q_{1} \frac{\partial}{\partial \zeta}$ and $H_{2}=$ $-q_{2} \frac{\partial}{\partial \zeta}$, we have $\tau_{1}=\frac{\partial}{\partial \xi_{1}}$ and $\tau_{2}=\frac{\partial}{\partial \xi_{2}}$ which yields $\left[\tau_{1}, \tau_{2}\right]=0$. 
With the deduced $r$ independent vector fields $\bar{\tau}_{i}$ for $1 \leq i \leq r$, we can then define the following matrix:

$$
\begin{aligned}
\Lambda_{1}= & \left(\theta_{j}\left(\bar{\tau}_{i}\right)\right)_{1 \leq i, j \leq r}=\left(\begin{array}{c}
\theta_{1} \\
\vdots \\
\theta_{r}
\end{array}\right)\left(\bar{\tau}_{1}, \cdots, \bar{\tau}_{r}\right) \\
= & \left(\begin{array}{cccc}
0 & \cdots & 0 & 1 \\
\vdots & \cdots & 1 & \lambda_{2, r} \\
0 & \cdots & \lambda_{r-1, r-1} & \lambda_{r-1, r} \\
1 & \cdots & \lambda_{r, r-1} & \lambda_{r, r}
\end{array}\right)
\end{aligned}
$$

with $\lambda_{i, j}=\theta_{i}\left(\bar{\tau}_{j}\right)$ for $1 \leq i, j \leq r$. Since it is invertible, we can define the following 1 -forms:

$$
\left(\begin{array}{c}
\omega_{1} \\
\vdots \\
\omega_{r}
\end{array}\right)=\Lambda_{1}^{-1}\left[\theta_{1}, \cdots, \theta_{r}\right]^{T}
$$

A straightforward calculation shows that

$$
\begin{aligned}
& \omega_{r}=\theta_{1} \\
& \omega_{r-k}=\left(\theta_{k+1}-\sum_{i=r-k+1}^{n} \lambda_{k+1, i} \omega_{i}\right) \text { for } 1 \leq k \leq r-1
\end{aligned}
$$

which are linear combination of the 1 -forms $\theta_{i}$ for $1 \leq i \leq r$. Then it is easy to verify that the deduced 1 -forms $\omega_{i}$ for $1 \leq i \leq$ $r$ have the following properties.

Lemma 5. 1) the annihilator of 1-forms $\omega_{k}$ for $1 \leq k \leq r$ is equal $\Delta^{\perp}$ defined in (9).

2) the 1-forms $\omega_{k}$ for $1 \leq k \leq r$ are well defined such that they are independent of the choice of $\bar{\tau}_{i}$ modulo $\Delta^{\perp}$.

3) for $1 \leq i, k \leq r, \omega_{k}\left(\bar{\tau}_{i}\right)=\delta_{k}^{i}$ where $\delta_{k}^{i}$ represents the Kronecker delta, i.e. $\delta_{k}^{i}=1$ if $k=i$, otherwise $\delta_{k}^{i}=0$.

Proof. The above properties are just the obvious results of (15) and (16), thus the proof is omitted.

The above lemma shows that, for any choice solution of (10), the constructed family of vector fields always yields the same 1 -form $\omega_{k}$ for $1 \leq k \leq r$, which allows us to state the following theorem.

Theorem 1. There exists a local diffeomorphism $\left(\xi^{T}, \zeta^{T}\right)^{T}=$ $\phi(x)$ on $\mathscr{X}$ which transforms system (1)-(2) into the proposed normal form (7), if and only if the following conditions are fulfilled:

1) there exists a family of vector fields $\tau_{i}=\bar{\tau}_{i}$ modulo $\Delta^{\perp}$ such that $\left[\tau_{i}, \tau_{l}\right]=0$ for $1 \leq i \leq r$ and $1 \leq l \leq r$;

2) $\left[\tau_{i}, \tau_{j}\right]=0$ for $1 \leq i \leq r$ and $r+1 \leq j \leq n$ where $\tau_{j}$ was defined in P2 of Lemma 1;

3) $\left[\tau_{i}, g_{k}\right] \in \Delta^{\perp}$ for $1 \leq i \leq n, i \neq r$ and $1 \leq k \leq m$.

If the above conditions are fulfilled, then the diffeomorphism $\phi$ is locally determined by its differential $\phi_{*}:=d \phi=\omega$, i.e. $\phi(x)=\int_{\gamma} \omega$, where $\gamma$ is any path on $\mathscr{X}$ with $\gamma(0)=0$ and $\gamma(1)=x$.
Proof.

Necessity: To show those conditions are necessary, let us assume that there exists a local diffeomorphism $\left(\xi^{T}, \zeta^{T}\right)^{T}=\phi(x)$ on $\mathscr{X}$ which transforms (1)-(2) into the proposed form (7).

Note $\omega=d \phi$, thus $\omega_{i}$ for $1 \leq i \leq r$ are the pullbacks of $d \xi_{i}$, i.e. ${ }^{2} \omega_{i}=\phi^{*}(d \xi)=d \phi_{i}$. For the same reason, $\omega_{j}$ for $r+1 \leq$ $j \leq n$ are the pullbacks of $d \zeta_{i}$ for $1 \leq i \leq n-r$ with $\omega_{j}=d \phi_{j}$.

In the following, we will firstly check that the conditions $i$ ), ii) and iii) of Theorem 1 are satisfied for the transformed normal form (7), then we will use the property of pullback to prove the necessity for the original system (1)-(2). In order to avoid the ambiguities, we add the superscript ' $n f$ ' for those calculations made for the transformed normal form (7), otherwise it is for the original system (1)-(2).

For the transformed normal form (7), following the same procedure presented in Section 3, we have $\theta_{1}^{n f}=d \xi_{r}$ and $\theta_{i}^{n f}=d \xi_{r-i+1}+\sum_{j=1}^{i-1} l_{j} \theta_{j}^{n f}$ for $2 \leq i \leq r$, which yields $\Delta^{n f}{ }^{\perp}=$ span $\left\{\frac{\partial}{\partial \zeta_{1}}, \cdots, \frac{\partial}{\partial \zeta_{n-r}}\right\}$. Then, we have $\bar{\tau}_{1}^{n f}=\frac{\partial}{\partial \xi_{1}}+H_{1}^{n f}$ with $H_{1}^{n f} \in \Delta^{n f}{ }^{\perp}$, based on which we can construct the rest vector fields: $\bar{\tau}_{i}^{n f}=\frac{\partial}{\partial \xi_{i}}+H_{i}^{n f}$ with $H_{i}^{n f} \in \Delta^{n f} \perp$ for $2 \leq i \leq r$. It is clear that there exists a family of vector fields $\tau_{i}^{n f}=\frac{\partial}{\partial \xi_{i}}=$ $\bar{\tau}_{i}^{n f}$ modulo $\Delta^{n f}{ }^{\perp}$ such that $\left[\tau_{i}^{n f}, \tau_{l}^{n f}\right]=0$ for $1 \leq i \leq r$ and $1 \leq l \leq r$.

Moreover, since $\tau_{j}^{n f}$ for $r+1 \leq j \leq n$ should be commutative and span $\Delta^{n f}{ }^{\perp}$ defined above, without loss of generalities, we can choose $\tau_{j}^{n f}=\frac{\partial}{\partial \zeta_{j-r}}$, with which we can prove that $\left[\tau_{i}^{n f}, \tau_{j}^{n f}\right]=0$ for $1 \leq i \leq r$ and $r+1 \leq j \leq n$.

Considering now the term in front of the input in (7). It is clear that $\left[\tau_{i}^{n f},\left[\begin{array}{c}\alpha_{k}^{1}(y) \\ \alpha_{k}^{2}(\xi, \zeta)\end{array}\right]\right] \in \Delta^{n f \perp}$ for $1 \leq i \leq n, i \neq r$ and $1 \leq k \leq m$.

Finally we proved that if there exist a local diffeomorphism $\left(\xi^{T}, \zeta^{T}\right)^{T}=\phi(x)$ on $\mathscr{X}$ which transforms (1)-(2) into (7), then there exists a family of vector fields $\tau_{i}^{n f}$ for $1 \leq i \leq n$ such that the condition $i$ ), ii) and $i i i$ ) are satisfied for the transformed normal form (7).

Since $\omega=d \phi$ are the pullbacks of $[d \xi, d \zeta]^{T}$, due to the fact that $\tau_{i}^{n f}=\frac{\partial}{\partial \xi_{i}}$ for $1 \leq i \leq r$ and $\tau_{j}^{n f}=\frac{\partial}{\partial \zeta_{j-r}}$ for $r+1 \leq j \leq n$, by defining

$$
\tau_{i}=\phi_{*}^{-1}\left(\tau_{i}^{n f}\right), \text { for } 1 \leq i \leq n
$$

we can conclude that if there exists a local diffeomorphism $\left(\xi^{T}, \zeta^{T}\right)^{T}=\phi(x)$ on $\mathscr{X}$ which transforms system (1)-(2) into the proposed normal form (7), then there exists a family of vector fields $\tau_{i}$ for $1 \leq i \leq n$ defined in (17) such that the condition $i$ ), ii) and iii) are necessary to be satisfied for the original system (1)-(2).

Sufficiency: Suppose that the conditions 1), 2) and 3) in Theorem 1 are satisfied, and we will prove that there exists a local

\footnotetext{
${ }^{2}$ Do not confuse the differential (Jacobian) of $\phi: \phi_{*}$ where the star is subscript with the pullback: $\phi^{*}$ where the star is superscript.
} 
diffeomorphism.

As $\left[\tau_{i}, \tau_{j}\right]=0$ for $1 \leq i \leq n$ and $1 \leq j \leq n$, then there exist $n$ independent 1 -forms $\bar{\omega}_{i}$ for $1 \leq i \leq n$ such that $\bar{\omega}_{i}\left(\tau_{j}\right)=\delta_{i}^{j}$ for $1 \leq j \leq n$. The following shows how to determine those 1-forms.

Firstly, since $\tau_{k}=\bar{\tau}_{k}$ modulo $\Delta^{\perp}$, according to Lemma 5, we have $\omega_{l}\left(\tau_{k}\right)=\delta_{l}^{k}$ for $1 \leq l, k \leq r$ where $\omega_{l}$ was given in (15), which implies that $\bar{\omega}_{l}=\omega_{l}$ for $1 \leq l \leq r$. Secondly, for $r+1 \leq$ $i \leq n$, since we have $\bar{\omega}_{i}\left(\tau_{j}\right)=\delta_{i}^{j}$ where $\tau_{j}$ was defined in P2 of Lemma 1 for $1 \leq j \leq n$, and this will uniquely determine the rest $(n-r) 1$-forms $\bar{\omega}_{i}$, noted as

$$
\omega_{i}=\bar{\omega}_{i} \text { such that } \bar{\omega}_{i}\left(\tau_{j}\right)=\delta_{i}^{j} \text { for } r+1 \leq i \leq n
$$

Finally, we uniquely determine the 1 -forms $\bar{\omega}=\omega=$ $\left[\omega_{1}, \cdots, \omega_{n}\right]^{T}$.

For those determined 1-forms, for $1 \leq k \leq n$ we have (see the beginning of the proof for Lemma 1):

$$
d \omega_{k}\left(\tau_{i}, \tau_{j}\right)=L_{\tau_{i}} \omega_{k}\left(\tau_{j}\right)-L_{\tau_{j}} \omega_{k}\left(\tau_{i}\right)-\omega_{k}\left(\left[\tau_{i}, \tau_{j}\right]\right) .
$$

As $\omega_{k}\left(\tau_{j}\right)$ and $\omega_{k}\left(\tau_{i}\right)$ are both constant (either 0 or 1 depending on the indices), then one has $d \omega_{k}\left(\tau_{i}, \tau_{j}\right)=-\omega_{k}\left(\left[\tau_{i}, \tau_{j}\right]\right)$ which implies the equivalence between $d \omega_{k}=0$ and $\left[\tau_{i}, \tau_{j}\right]=0$, since $\omega_{k}$ and $\tau_{i}$ are dual and independent. Therefore, the condition ii) implies the deduced $\omega$ defined in (15) and (18) is closed, i.e. $d \omega=0$. Then, thanks to Poincar's lemma, there exists a local diffeomorphism $\phi$ such that $\phi_{*}=\omega$, where $\phi_{*}$ represents the Jacobian of $\phi$.

In what follows, we will prove that $\phi$ deduced via $\phi_{*}=\omega$ is exactly the sought diffeomorphism which transforms system (1)-(2) into the proposed normal form (7).

For this, let us firstly show how it transforms $f$ in (1). Setting $\left(\xi^{T}, \zeta^{T}\right)^{T}=\phi(x)$, since $\phi_{*}=\omega$, then we have $\phi_{*}\left(\tau_{i}\right)=$ $\omega\left(\tau_{i}\right)=\frac{\partial}{\partial \xi_{i}}$ for $1 \leq i \leq r$ and $\phi_{*}\left(\tau_{j}\right)=\omega\left(\tau_{j}\right)=\frac{\partial}{\partial \zeta_{j}}$ for $r+1 \leq i \leq n$. Moreover, according to the third property of Lemma 5, for any $H \in \Delta^{\perp}$, we have $\omega_{i}(H)=0$ for $1 \leq i \leq r$ and $\omega_{j}(H) \in \operatorname{span}\left\{\frac{\partial}{\partial \zeta_{1}}, \cdots, \frac{\partial}{\partial \zeta_{n-r}}\right\}$ for $r+1 \leq j \leq n$ because $\phi_{*}\left(\tau_{j}\right)=\omega\left(\tau_{j}\right)=\frac{\partial}{\partial \zeta_{j}}$ and $\tau_{j}$ span the distribution $\Delta^{\perp}$. Thus, for any $H \in \Delta^{\perp}$ we have $\phi_{*}(H)=\omega(H) \in \operatorname{span}\left\{\frac{\partial}{\partial \zeta_{1}}, \cdots, \frac{\partial}{\partial \zeta_{n-r}}\right\}$. Now, let us consider the following two cases:

- for $1 \leq i \leq r-1$, we have $\frac{\partial}{\partial \xi_{i}} \phi_{*}(f)=\left[\frac{\partial}{\partial \xi_{i}}, \phi_{*}(f)\right]=$ $\left[\phi_{*}\left(\tau_{i}\right), \phi_{*}(f)\right]=\phi_{*}\left[\tau_{i}, f\right]=\phi_{*}\left(\tau_{i+1}+H_{i}\right)$ for some $H_{i} \in$ $\Delta^{\perp}$. Thus we have

$$
\frac{\partial}{\partial \xi_{i}} \phi_{*}(f)=\phi_{*}\left(\tau_{i+1}\right)+\phi_{*}\left(H_{i}\right)=\frac{\partial}{\partial \xi_{i+1}}+\phi_{*}\left(H_{i}\right) ;
$$

- for $r+1 \leq j \leq n$, we have $\frac{\partial}{\partial \zeta_{j}} \phi_{*}(f)=\left[\frac{\partial}{\partial \zeta_{j}}, \phi_{*}(f)\right]=$ $\left[\phi_{*}\left(\tau_{j}\right), \phi_{*}(f)\right]=\phi_{*}\left[\tau_{j}, f\right]$. Since $\tau_{j}$ span the distribution $\Delta^{\perp}$ which is $f$-invariant (see $\mathrm{P} 3$ of Lemma 1 ), then there exist $H_{j} \in \Delta^{\perp}$ such that $H_{j}=\left[\tau_{j}, f\right]$. Then we have

$$
\frac{\partial}{\partial \zeta_{j}} \phi_{*}(f)=\phi_{*}\left(H_{j}\right)
$$

Since for any $H \in \Delta^{\perp}$ we have $\phi_{*}(H) \in$ span $\left\{\frac{\partial}{\partial \zeta_{1}}, \cdots, \frac{\partial}{\partial \zeta_{n-r}}\right\}$, thus, by integrating (19) and (20), we obtain $\phi_{*}(f)=\left[\begin{array}{c}A \xi+\beta(y) \\ \eta(\xi, \zeta)\end{array}\right]$.

Consider now the term $g$ in (1). If the condition iii) of Theorem 1 is satisfied, i.e. $\left[\tau_{i}, g_{k}\right] \in \Delta^{\perp}$ for $1 \leq i \leq n, i \neq r$ and $1 \leq k \leq m$, then we have $\phi_{*}\left(\left[\tau_{i}, g_{k}\right]\right) \in \operatorname{span}\left\{\frac{\partial}{\partial \zeta_{1}}, \cdots, \frac{\partial}{\partial \zeta_{n-r}}\right\}$. By applying the same argument, for $1 \leq k \leq m$ let us consider the following two cases:

- for $1 \leq i \leq r-1$, we have $\frac{\partial}{\partial \xi_{i}} \phi_{*}\left(g_{k}\right)=\left[\frac{\partial}{\partial \xi_{i}}, \phi_{*}\left(g_{k}\right)\right]=$ $\left[\phi_{*}\left(\tau_{i}\right), \phi_{*}\left(g_{k}\right)\right]=\phi_{*}\left[\tau_{i}, g_{k}\right]$;

- for $r+1 \leq j \leq n$, for the same reason we have $\frac{\partial}{\partial \zeta_{j}} \phi_{*}\left(g_{k}\right)=$ $\phi_{*}\left[\tau_{j}, g_{k}\right]$

Since $\phi_{*}\left(\left[\tau_{i}, g_{k}\right]\right) \in \operatorname{span}\left\{\frac{\partial}{\partial \zeta_{1}}, \cdots, \frac{\partial}{\partial \zeta_{n-r}}\right\}$ for $1 \leq i \leq n, i \neq r$ and $1 \leq k \leq m$, the integration of the above two equations yields $\phi_{*}\left(g_{k}\right)=\left[\begin{array}{c}\alpha_{k}^{1}(y) \\ \alpha_{k}^{2}(\xi, \zeta)\end{array}\right]$ for $1 \leq k \leq m$.

Following the same argument, one can prove as well $\left[\tau_{i}, g_{k}\right] \in$ modulo $\Delta^{\perp}$ for $1 \leq i \leq n, i \neq r$ and $1 \leq k \leq m$.

The rest concerns the deduction of the local diffeomorphism. Since $\omega=d \phi$ on $\mathscr{X}$, then the diffeomorphism can be determined just by integration of $\omega$, i.e. $\phi(x)=\int_{\gamma} \omega$ where $\gamma$ is any path on $\mathscr{X}$ with $\gamma(0)=0$ and $\gamma(1)=x$.

For any choice of $\bar{\tau}_{1}$ satisfying (10) with the associated family of vector fields $\bar{\tau}_{i}$ for $2 \leq i \leq r$, Theorem 1 needs to seek a new family of vector fields $\tau_{i}=\bar{\tau}_{i}$ modulo $\Delta^{\perp}$ such that $\left[\tau_{i}, \tau_{l}\right]=0$ for $1 \leq i \leq r$ and $1 \leq l \leq r$. Thanks to Lemma 4 , we can remove at the beginning all terms in the directions of the basis of $\Delta^{\perp}$ in $\bar{\tau}_{1}$, noted as $\tau_{1}$. It is important to emphasize that this elimination will yield a unique $\tau_{1}$ for any solution of (10). Then, we can iteratively eliminate all terms in the directions of the basis of $\Delta^{\perp}$ in $\bar{\tau}_{i}$, which gives the following simple procedure to calculate $\tau_{i}$ for $1 \leq i \leq n$ :

Procedure 1. For the nonlinear system (1)-(2), the family of vector fields $\tau_{i}$ for $1 \leq i \leq n$ can be simply computed via the following steps:

Step 1: Compute the observability 1-forms $\theta_{i}$ for $1 \leq i \leq r$;

Step 2: Determine $\Delta^{\perp}$ and seek a commutative basis $\left\{\tau_{r+1}, \cdots, \tau_{n}\right\}$ that spans $\Delta^{\perp}$

Step 3: Choose any solution $\bar{\tau}_{1}$ of $(10)$, then eliminate all its terms in the directions of $\left\{\tau_{r+1}, \cdots, \tau_{n}\right\}$ which yields $\tau_{1}$

Step 4: Iteratively, for $2 \leq i \leq r$, compute $\left[\tau_{i-1}, f\right]$ in which eliminate all terms in the directions of $\left\{\tau_{r+1}, \cdots, \tau_{n}\right\}$ which gives $\tau_{i}$.

Remark 3. Like most of existing results by using the differential geometric method, such as the pioneering works in Krener and Isidori (1983); Krener and Respondek (1985); Respondek et al. (2004); Jo and Seo (2002), the proposed results in this paper characterize only the solvability of the problem. It is worthy noting that the construction of such family of commutative vector fields is still quite challenging. 
The following example is to highlight Theorem 1 by following Procedure 1.

Example 2. Let us consider the following nonlinear system:

$$
\left\{\begin{array}{l}
\dot{x}_{1}=-x_{3}^{2}+x_{1}^{3} x_{3}-\frac{1}{2} x_{3}^{3}+x_{2}^{5} \\
\dot{x}_{2}=x_{1}-\frac{1}{2} x_{3}^{2} \\
\dot{x}_{3}=-x_{3}+x_{1}^{3}-\frac{1}{2} x_{3}^{2} \\
y=x_{2}
\end{array}\right.
$$

Step 1:

A simple calculation gives $\operatorname{rank}\left\{d h, d L_{f} h, d L_{f}^{2} h\right\}=2$, thus $r=$ 2 , and one has $\theta_{1}=d x_{2}$ and $\theta_{2}=d x_{1}-x_{3} d x_{3}$. since $d L_{f}^{2} h=$ $5 x_{2}^{4} \theta_{1}$.

\section{Step 2:}

Then one obtains $\Delta=\operatorname{span}\left\{\theta_{1}, \theta_{2}\right\}$, and

$$
\Delta^{\perp}=\operatorname{span}\left\{x_{3} \frac{\partial}{\partial x_{1}}+\frac{\partial}{\partial x_{3}}\right\}
$$

Since $\tau_{3}$ should be a commutative basis of $\Delta^{\perp}$, thus one has $\tau_{3}=x_{3} \frac{\partial}{\partial x_{1}}+\frac{\partial}{\partial x_{3}}$.

Step 3:

By solving equation (10), one obtains $\bar{\tau}_{1}=\frac{\partial}{\partial x_{1}}+q_{1}(x) \tau_{3}$. Following Procedure 1, by eliminating all terms in $\bar{\tau}_{1}$ in the direction of $\tau_{3}$, we have $\tau_{1}=\frac{\partial}{\partial x_{1}}$.

\section{Step 4:}

Then we can calculate: $\left[\tau_{1}, f\right]=\frac{\partial}{\partial x_{2}}+3 x_{1}^{2} \tau_{3}$, for which the elimination of all terms in the direction of $\tau_{3}$ yields $\tau_{2}=\frac{\partial}{\partial x_{2}}$.

Finally Procedure 1 enables us to easily get the following vector fields:

$$
\tau_{1}=\frac{\partial}{\partial x_{1}}, \tau_{2}=\frac{\partial}{\partial x_{2}}, \tau_{3}=x_{3} \frac{\partial}{\partial x_{1}}+\frac{\partial}{\partial x_{3}}
$$

with which it is easy to check that all conditions of Theorem 1 are satisfied, thus there exists a diffeomorphism which can transform the studied example into the proposed form (7).

For the deduction of the diffeomorphism, we use the obtained vector field $\tau=\left[\tau_{1}, \tau_{2}, \tau_{3}\right]$ which gives:

$$
\Lambda_{1}=\left[\begin{array}{l}
\theta_{1} \\
\theta_{2}
\end{array}\right]\left(\tau_{1}, \tau_{2}\right)=\left(\begin{array}{ll}
0 & 1 \\
1 & 0
\end{array}\right)
$$

thus one has

$$
\left(\begin{array}{l}
\omega_{1} \\
\omega_{2}
\end{array}\right)=\Lambda_{1}^{-1}\left(\begin{array}{c}
\theta_{1} \\
\theta_{2}
\end{array}\right)=\left(\begin{array}{c}
d \xi_{1} \\
d \xi_{2}
\end{array}\right)=d\left(\begin{array}{c}
x_{1}-\frac{1}{2} x_{3}^{2} \\
x_{2}
\end{array}\right)
$$

Following (18), one can solve the following equation:

$$
\omega_{3}\left(\frac{\partial}{\partial x_{1}}, \frac{\partial}{\partial x_{2}}, \frac{\partial}{\partial x_{3}}+x_{3} \frac{\partial}{\partial x_{1}}\right)=(0,0,1)
$$

which uniquely determines $\omega_{3}=d x_{3}$. Finally, one gets the following diffeomorphism $\phi(x)=\left(\begin{array}{c}x_{1}-\frac{1}{2} x_{3}^{2} \\ x_{2} \\ x_{3}\end{array}\right)$ which transforms the studied system (21) into the following form

$$
\left\{\begin{array}{l}
\dot{\xi}_{1}=0 \\
\dot{\xi}_{2}=\xi_{1} \\
\dot{\zeta}_{1}=\xi_{1}^{3}-\xi_{3}+\frac{\xi_{3}^{2}}{2}\left(3 \xi_{1}^{2}+\frac{3}{2} \xi_{1} \xi_{3}^{2}+\frac{1}{4} \xi_{3}^{4}-1\right) \\
y=\xi_{2}
\end{array}\right.
$$

\section{Diffeomorphism on the output}

This section deals with the case when the conditions of Theorem 1 are not fulfilled. Let us remark that the deduced diffeomorphism $\phi(x)$ in Section 3 does not modify the output. This is due to the fact that $\theta_{r}\left(\bar{\tau}_{1}\right)=1$. One way to relax this constraint is to seek a new vector field, noted as $\bar{\sigma}_{1}$, such that $\theta_{r}\left(\bar{\sigma}_{1}\right)$ becomes a function of the output, and this will introduce a diffeomorphism on the output. For that purpose, this section will modify the new vector field $\tau_{1}$ obtained in Section 3 by following Procedure 1 and construct a new family of commutative vector fields, with which the deduced diffeomorphism $\phi(x)$ will apply as well a change of coordinates on the output (see Krener and Respondek (1985); Respondek et al. (2004); Boutat and Busawon (2011)). Since this diffeomorphism will modify the output of the studied system (1)-(2), therefore the PONF (7) is adapted as follows:

$$
\left\{\begin{array}{l}
\dot{\xi}=A \xi+\beta(y)+\sum_{k=1}^{m} \alpha_{k}^{1}(y) u_{k} \\
\dot{\zeta}=\eta(\xi, \zeta)+\sum_{k=1}^{m} \alpha_{k}^{2}(\xi, \zeta) u_{k} \\
\bar{y}=\xi_{r}=\psi(y)
\end{array}\right.
$$

With the same procedure presented in Section 3, we can calculate the observability 1 -forms $\theta_{i}$ for $1 \leq i \leq r$ where $r$ is the rank of observability matrix, which defines the co-distribution $\Delta=\operatorname{span}\left\{\theta_{1}, \theta_{2}, \cdots, \theta_{r}\right\}$, and the kernel (or the annihilator) of the co-distribution $\Delta$, noted as $\Delta^{\perp}$.

By following Procedure 1 presented in Section 3, it is assumed that we have already obtained a family of vector fields $\tau_{j}$ for $r+1 \leq j \leq n$ which are commutative basis of $\Delta^{\perp}$, and $\tau_{i}$ for $1 \leq i \leq r$ which do not contain any component in the direction of $\tau_{j}$ for $r+1 \leq j \leq n$. In this section, it is assumed that there exist $1 \leq i, l \leq r$ such that $\left[\tau_{i}, \tau_{l}\right] \neq 0$, thus Theorem 1 cannot be applied. In order to introduce a change of coordinate for the output, let $s(y) \neq 0$ be a function of the output which will be determined later. Then one can define a new vector field $\sigma_{1}$ from $\tau_{1}$ as follows:

$$
\bar{\sigma}_{1}=s(y) \tau_{1}
$$

By induction, one can define the following new vector fields $\bar{\sigma}_{i}=\left[\bar{\sigma}_{i-1}, f\right]$ modulo $\Delta^{\perp}$, for $2 \leq i \leq r$. A straightforward calculation gives

$$
\bar{\sigma}_{i}=\sum_{k=1}^{i}(-1)^{i-k} C_{i-1}^{k-1} L_{f}^{i-k} s(y) \tau_{k} \text { modulo } \Delta^{\perp}
$$


where $C_{i-1}^{k-1}$ is the binomial coefficients and $L_{f}^{0} s(y)=s(y)$.

For the same reason stated in Procedure 1, by eliminating all terms in the directions of $\tau_{j}$ for $r+1 \leq j \leq n$ (the commutative basis of $\Delta^{\perp}$ ) we obtain:

$$
\sigma_{i}=\sum_{k=1}^{i}(-1)^{i-k} C_{i-1}^{k-1} L_{f}^{i-k} s(y) \tau_{k}, \text { for } 1 \leq i \leq r
$$

For the sake of completeness, we simply note

$$
\sigma_{j}=\tau_{j}, \text { for } r+1 \leq j \leq n
$$

where $\left\{\tau_{r+1}, \cdots, \tau_{n}\right\}$ are commutative basis of $\Delta^{\perp}$, obtained by following Procedure 1 presented in Section 3.

We can then define the following matrix:

$$
\tilde{\Lambda}_{1}=\left(\begin{array}{l}
\theta_{1} \\
\vdots \\
\theta_{r}
\end{array}\right)\left(\sigma_{1}, \cdots, \sigma_{r}\right)=\left(\begin{array}{cccc}
0 & \cdots & 0 & s(y) \\
\vdots & \cdots & s(y) & * \\
0 & \cdots & * & * \\
s(y) & \cdots & * & *
\end{array}\right)
$$

Since $s(y) \neq 0$, thus it is invertible, and we can define the following 1-forms:

$$
\left(\begin{array}{c}
\tilde{\omega}_{1} \\
\vdots \\
\tilde{\omega}_{r}
\end{array}\right)=\tilde{\Lambda}_{1}^{-1}\left[\theta_{1}, \cdots, \theta_{r}\right]^{T}
$$

and using the same method in Section 3 we can uniquely determine $\widetilde{\omega}_{i}$ for $r+1 \leq i \leq n$ by solving the following equations:

$$
\tilde{\omega}_{i}\left(\sigma_{j}\right)=\delta_{i}^{j}
$$

for $1 \leq j \leq n$. Finally we have the following theorem.

Theorem 2. There exists a local diffeomorphism $\left(\xi^{T}, \zeta^{T}\right)^{T}=$ $\phi(x)$ on $\mathscr{X}$ which transforms system (1)-(2) into the PONF (22) if and only if the following conditions are fulfilled:

1) $\left[\sigma_{i}, \sigma_{l}\right]=0$ for $1 \leq i \leq r$ and $1 \leq l \leq r$ where $\sigma_{i}$ defined in (25);

2) $\left[\sigma_{i}, \sigma_{j}\right]=0$ for $1 \leq i \leq r$ and $r+1 \leq j \leq n$ where $\sigma_{j}$ defined in (26);

3) $\left[\sigma_{i}, g_{k}\right] \in \Delta^{\perp}$ for $1 \leq i \leq n, i \neq r$ and $1 \leq k \leq m$.

If the above conditions are fulfilled, then the diffeomorphism $\phi$ is locally determined by its differential $\phi_{*}:=d \phi=\tilde{\omega}$, i.e. $\phi(x)=\int_{\gamma} \tilde{\omega}$, where $\gamma$ is any path on $\mathscr{X}$ with $\gamma(0)=0$ and $\gamma(1)=x$. Moreover, one has $\xi_{r}=\bar{y}=\psi(y)$ where $\psi(y)=$ $\int_{0}^{y} \frac{1}{s(c)} d c$ is a change of coordinates on the output.

\section{Proof.}

The proof of Theorem 2 is similar to the proof of Theorem 1 , thus the following just gives a sketch to determine $s(y)$ and some different elements with respect to the result stated in Boutat and Busawon (2011).
According to (23), we define $\sigma_{1}=s(y) \tau_{1}$ where $\tau_{1}$ is obtained in Section 3 by eliminating all terms in the directions of $\tau_{j}$ for $r+1 \leq j \leq n$. By induction, we have $\sigma_{i}=$ $\left[\sigma_{i-1}, f\right]$ modulo $\Delta^{\perp}$, for $2 \leq i \leq r$. Then one can deduce:

$$
\begin{aligned}
{\left[\sigma_{1}, \sigma_{i}\right]=} & \sum_{k=1}^{i}(-1)^{i-k} C_{i-1}^{k-1} L_{f}^{i-k} s(y)\left[\sigma_{1}, \tau_{k}\right] \\
= & \sum_{k=1}^{i}(-1)^{i-k} C_{i-1}^{k-1} L_{f}^{i-k} s(y)\left[s(y)\left[\tau_{1}, \tau_{k}\right]-L_{\tau_{k}} s(y) \tau_{1}\right] \\
& +\sum_{k=1}^{i}(-1)^{i-k} C_{i-1}^{k-1} L_{\sigma_{1}} L_{f}^{i-k} s(y) \tau_{k}
\end{aligned}
$$

Imposing $\left[\sigma_{1}, \sigma_{i}\right]=0$, this enables us to determine $s(y)$. More details on this issue can be founded in Boutat and $\mathrm{Bu}-$ sawon (2011). After having deduced $s(y)$, the rest of the proof for Theorem 2 is similar to the proof of Theorem 1 .

Example 3. Let us consider the well-known SIR (Susceptible, Infected, Removed) epidemic model describing the contagious disease propagation Raggett (1982); Feng and Garrido (2008) as follows:

$$
\left\{\begin{array}{l}
\dot{S}=-\beta S I \\
\dot{I}=\beta S I-\gamma I \\
\dot{R}=\gamma I \\
y=I
\end{array}\right.
$$

where $S$ denotes the suspected population, I denotes the infected and $R$ denotes the removed population and the total population.

By using the same notations introduced in Section 3, a simple calculation gives $\theta_{1}=d I$ and $\theta_{2}=\beta I d S+(\beta S-\gamma) d I$, and we have $\Delta=\operatorname{span}\{d I, \beta I d S+(\beta S-\gamma) d I\}$ which gives $\Delta^{\perp}=$ $\operatorname{span}\left\{\frac{\partial}{\partial R}\right\}$.

\section{Calculation of $\bar{\tau}$ :}

Following the procedure proposed in Krener and Isidori (1983), we obtain the following vector fields:

$$
\begin{aligned}
& \bar{\tau}_{1}=\frac{1}{\beta I} \frac{\partial}{\partial S}+q_{1} \frac{\partial}{\partial R} \\
& \bar{\tau}_{2}=\left[\bar{\tau}_{1}, f\right]=\frac{\partial}{\partial I}+\frac{\beta S-\gamma-\beta I}{\beta I} \frac{\partial}{\partial S}+q_{2} \frac{\partial}{\partial R}
\end{aligned}
$$

with $q_{1}(S, I, R)$ is any function and $q_{2}=-L_{f} q_{1}$, which gives $\left[\bar{\tau}_{1}, \bar{\tau}_{2}\right] \neq 0$. Therefore the conditions stated in Jo and Seo (2002) are not fulfilled.

\section{Calculation of $\tau$ :}

Applying Procedure 1 proposed in Section 3, we obtain the following new vector fields:

$$
\begin{aligned}
\tau_{1} & =\frac{1}{\beta I} \frac{\partial}{\partial S} \\
\tau_{2} & =\frac{\partial}{\partial I}+\frac{\beta S-\gamma-\beta I}{\beta I} \frac{\partial}{\partial S} \\
\tau_{3} & =\frac{\partial}{\partial R}
\end{aligned}
$$

It is obvious that $\left[\tau_{1}, \tau_{2}\right] \neq 0$, which implies that the conditions of Theorem 1 are not fulfilled.

\section{Calculation of $\sigma$ :}

In order to construct a new family of commutative vector fields by introducing a change of coordinates on the output, the 
method proposed in Boutat and Busawon (2011) is used to deduce a non-zero output function $s(y)$. For this, set $\sigma_{1}=s(y) \tau_{1}$. According to (25) one has $\sigma_{2}=s(y) \tau_{2}-s^{\prime}(y)(\beta S I-\gamma I) \tau_{1}$. A straightforward calculation gives:

$$
\left[\sigma_{1}, \sigma_{2}\right]=\left(\frac{2 s^{2}(y)}{I}-2 s(y) s^{\prime}(y)\right) \tau_{1}
$$

thus $\left[\sigma_{1}, \sigma_{2}\right]=0$ if and only if the function $s(y)$ fulfills the following differential equation:

$$
\frac{s(y)}{y}-\frac{d s(y)}{d y}=0
$$

which motivates us to choose $s(y)=y=I$. Then one has:

$$
\sigma_{1}=\frac{1}{\beta} \frac{\partial}{\partial S}, \sigma_{2}=-I \frac{\partial}{\partial S}+I \frac{\partial}{\partial I}, \sigma_{3}=\frac{\partial}{\partial R}
$$

with which one has

$$
\left[\sigma_{1}, \sigma_{2}\right]=\left[\sigma_{1}, \sigma_{3}\right]=\left[\sigma_{2}, \sigma_{3}\right]=0
$$

thus the first two conditions of Theorem 2 are satisfied. As the SIR model does not contain any input, then the third condition of Theorem 2 does not need to be verified.

Since

$$
\tilde{\Lambda}_{1}=\left[\begin{array}{l}
\theta_{1} \\
\theta_{2}
\end{array}\right]\left(\sigma_{1}, \sigma_{2}\right)=\left(\begin{array}{cc}
0 & I \\
I & -\beta I^{2}+(-\gamma+S \beta) I
\end{array}\right)
$$

which yields

$$
\left(\begin{array}{c}
\tilde{\omega}_{1} \\
\tilde{\omega}_{2}
\end{array}\right)=\tilde{\Lambda}^{-1}\left(\begin{array}{c}
\theta_{1} \\
\theta_{2}
\end{array}\right)=\left(\begin{array}{c}
d \xi_{1} \\
d \xi_{2}
\end{array}\right)=d\left(\begin{array}{c}
\beta(S+I) \\
\ln I
\end{array}\right)
$$

Following (29), one can solve the following equation:

$$
\tilde{\omega}_{3}\left(\sigma_{1}, \sigma_{2}, \sigma_{3}\right)=\tilde{\omega}_{3}\left(\frac{1}{\beta} \frac{\partial}{\partial S},-I \frac{\partial}{\partial S}+I \frac{\partial}{\partial I}, \frac{\partial}{\partial R}\right)=(0,0,1)
$$

which uniquely determines $\tilde{\omega}_{3}=d R$. Therefore, the local diffeomorphism is given as follows $\phi(x)=[\beta(S+I), \ln I, R]^{T}$, which transforms the SIR model into the following form:

$$
\left\{\begin{array}{l}
\dot{\xi}_{1}=-\beta \gamma e^{\bar{y}} \\
\dot{\xi}_{2}=\xi_{1}-\beta e^{\bar{y}}-\gamma \\
\dot{\zeta}_{1}=\gamma e^{\bar{y}} \\
\bar{y}=C \phi=\ln I
\end{array}\right.
$$

\section{Conclusion}

This paper deals with partial observability problem of nonlinear system. Necessary and sufficient conditions are established to guarantee the existence of a local diffeomorphism transforming a class of nonlinear systems into the proposed partial observer normal form with and without a change of coordinates on the output. For the transformed system, a simple Luenberger observer can be designed to estimate the part of observable states. Examples are provided to illustrate the feasibility of the proposed results.

\section{Appendix}

\section{A.1 Proof of Lemma 1:}

Proof. Firstly, let us recall that the differential $d \pi$ of a differential 1-form $\pi$ yields a 2-form whose evaluation on two vector fields $X$ and $Y$ is given by:

$$
d \pi(X, Y)=L_{X} \pi(Y)-L_{Y} \pi(X)-\pi([X, Y])
$$

If $\pi$ is closed, then $d \pi=0$ and the above formula becomes:

$$
\pi([X, Y])=L_{X} \pi(Y)-L_{Y} \pi(X)
$$

Denote $l_{X} \pi=\pi(X)$ as the interior product (or the contraction of a differential form with a vector field) of an 1-form $\pi$ by a vector field $X$ and recall that by definition the interior product of a function by a vector field is equal 0 . Then the interior product relates the exterior derivative and Lie derivative of differential forms by the following so-called Cartan's identity:

$$
L_{X}=d l_{X}+\iota_{X} d
$$

$\underline{\text { Proof of P1: }}$

According to the definition of the 1-forms $\theta_{i}=d L_{f}^{i-1} h$ for $1 \leq i \leq r$, thus $\theta_{i}$ are exact (then closed), which implies that for any two vector fields $H_{1} \in \Delta^{\perp}$ and $H_{2} \in \Delta^{\perp}$, we have $\left[H_{1}, H_{2}\right] \in \Delta^{\perp}$ due to the relation of (31). Thus $\Delta^{\perp}$ is involutive.

\section{Proof of P2:}

The second property is an obvious consequence of the first one thanks to the well-known Frobenius' Theorem (see Isidori (1995) for the details).

Proof of P3:

As $\theta_{i}$ for $1 \leq i \leq r$ are exact, thus for any vector field $H \in \Delta^{\perp}$, according to the equation (31) we have

$$
\theta_{i}([f, H])=L_{f} \theta_{i}(H)-L_{H} \theta_{i}(f)
$$

Since $H \in \Delta^{\perp}$, then $\theta_{i}(H)=0$ which implies that $L_{f} \theta_{i}(H)=0$. Therefore, $\theta_{i}([f, H])=0$ is equivalent to $L_{H} \theta_{i}(f)=0$. Moreover, based on the Cartan's identity (32), we have:

$$
L_{H} \theta_{i}(f)=d l_{H} \theta_{i}(f)+\iota_{H} d \theta_{i}(f)
$$

Due to the fact that $\theta_{i}(f)$ is a function, therefore its interior product by a vector field is 0 , thus $\imath_{H} \theta_{i}(f)=0$, which means $d_{l_{H}} \theta_{i}(f)=0$. So, the above equation becomes

$$
L_{H} \theta_{i}(f)=\iota_{H} d \theta_{i}(f)
$$

It reminds to show that $\iota_{H} d \theta_{i}(f)=0$. As $d \theta_{i}(f)=\theta_{i+1}$ for $1 \leq i \leq r-1$, then

$$
\iota_{H} d \theta_{i}(f)=\iota_{H} \theta_{i+1}=\theta_{i+1}(H)=0 .
$$

For $i=r$, we have $d \theta_{r}(f)=d L_{f}^{r+1} h$. Since it is assumed that $\operatorname{rank}\left\{d h, \cdots, d L_{f}^{n} h\right\}=r$, it means $d \theta_{r}(f)=\sum_{i=1}^{r} \gamma_{i} \theta_{i}$ which implies $\iota_{H} d \theta_{i}(f)=0$. 
Finally, we proved that, for any vector field $H \in \Delta^{\perp}$, one always has $\theta_{i}([f, H])=0$, which implies that $[f, H] \in \Delta^{\perp}$. Thus $\Delta^{\perp}$ is $f$-invariant.

\section{A.2 Proof of Lemma 2}

Proof. Since $\bar{\tau}_{1}$ and $\tilde{\tau}_{1}$ are both the solution of (10), then there exists an $H_{1} \in \Delta^{\perp}$ such that $\tilde{\tau}_{1}=\bar{\tau}_{1}+H_{1}$.

According to (11) and (12), we have

$$
\begin{aligned}
\tilde{\tau}_{2} & =\left[\tilde{\tau}_{1}, f\right]=\left[\bar{\tau}_{1}+H_{1}, f\right] \\
& =\left[\bar{\tau}_{1}, f\right]+\left[H_{1}, f\right]=\bar{\tau}_{2}+H_{2}
\end{aligned}
$$

where we applied the third property P3 of Lemma 1, i.e. $\left[H_{1}, f\right]=H_{2} \in \Delta^{\perp}$. By induction, we deduce that for $1 \leq i \leq r$ there exist $H_{i} \in \Delta^{\perp}$ such that $\tilde{\tau}_{i}=\bar{\tau}_{i}+H_{i}$.

\section{A.3 Proof of Lemma 3}

Proof. 1) For any $H \in \Delta^{\perp}$, as $d \theta_{k}=0$ since $\theta_{k}=d L_{f}^{k-1} h$ for $1 \leq k \leq r$ is closed, we have:

$$
\begin{aligned}
\theta_{k}\left(\left[\bar{\tau}_{i}, H\right]\right) & =L_{\bar{\tau}_{i}} \theta_{k}(H)-L_{H} \theta_{k}\left(\bar{\tau}_{i}\right) \\
& =-L_{H} \theta_{k}\left(\bar{\tau}_{i}\right)
\end{aligned}
$$

where we have applied $\theta_{k}(H)=0$ for $1 \leq k \leq r$ since $H \in \Delta^{\perp}$.

When $i=1$, then from (10) we have

$$
\theta_{k}\left(\bar{\tau}_{1}\right)= \begin{cases}0, & 1 \leq k \leq r-1 \\ 1, & k=r\end{cases}
$$

Thus, $\theta_{k}\left(\bar{\tau}_{1}\right)$ is constant, then its Lie derivative is 0 . Therefore, we have

$$
\theta_{k}\left(\left[\bar{\tau}_{1}, H\right]\right)=-L_{H} \theta_{k}\left(\bar{\tau}_{1}\right)=0
$$

which implies that $\left[\bar{\tau}_{1}, H\right] \in \Delta^{\perp}$.

For $i=2$, by applying Jacobi's identity we have

$$
\left[\bar{\tau}_{2}, H\right]=\left[\left[\bar{\tau}_{1}, f\right], H\right]=-\left[\left[H, \bar{\tau}_{1}\right], f\right]-\left[[H, f], \bar{\tau}_{1}\right]
$$

Since $\left[\bar{\tau}_{1}, H\right] \in \Delta^{\perp}$, and $[H, f] \in \Delta^{\perp}$ (P3 of Lemma 1), then we obtain $\left[\bar{\tau}_{2}, H\right] \in \Delta^{\perp}$.

Repeating the above procedure by using the Jacobi's identity, we can prove by induction that, for any $H \in \Delta^{\perp}$, we have:

$$
\left[\bar{\tau}_{i}, H\right] \in \Delta^{\perp}, \text { for } 1 \leq i \leq r .
$$

2) For any $H_{i} \in \Delta^{\perp}$ and $H_{j} \in \Delta^{\perp}$, it is clear that $\left[H_{i}, H_{j}\right] \in \Delta^{\perp}$. Then we have:

$$
\left[\bar{\tau}_{i}+H_{i}, \bar{\tau}_{j}+H_{j}\right]=\left[\bar{\tau}_{i}, \bar{\tau}_{j}\right]+\left[\bar{\tau}_{i}, H_{j}\right]+\left[H_{i}, \bar{\tau}_{j}\right]+\left[H_{i}, H_{j}\right]
$$

Since we have proved in the above that $\left[\bar{\tau}_{i}, H_{j}\right] \in \Delta^{\perp}$ and $\left[H_{i}, \bar{\tau}_{j}\right] \in \Delta^{\perp}$, with the fact that $\left[H_{i}, H_{j}\right] \in \Delta^{\perp}$, we get

$$
\left[\bar{\tau}_{i}+H_{i}, \bar{\tau}_{j}+H_{j}\right]=\left[\bar{\tau}_{i}, \bar{\tau}_{j}\right] \text { modulo } \Delta^{\perp}
$$

3) As we have proved that for any $H \in \Delta^{\perp}$ one has $\left[h, \bar{\tau}_{i}\right] \in \Delta^{\perp}$ for $1 \leq i \leq r$, due to the fact that $\tau_{j}$ for $r+1 \leq j \leq n$ are the basis of $\Delta^{\perp}$, therefore we have: $\left[\bar{\tau}_{i}, \tau_{j}\right] \in \Delta^{\perp}$.

\section{A.4 Proof of Lemma 4:}

Proof. Necessity: Suppose that there exist $H_{i} \in \Delta^{\perp}$ and $H_{j} \in$ $\Delta^{\perp}$, which determine $\tau_{i}=\bar{\tau}_{i}+H_{i}$ and $\tau_{j}=\bar{\tau}_{j}+H_{j}$ for $1 \leq i, j \leq$ $r$ such that $\left[\tau_{i}, \tau_{j}\right]=0$, then according to the second item of Lemma 3 we have

$$
0=\left[\tau_{i}, \tau_{j}\right]=\left[\bar{\tau}_{i}, \bar{\tau}_{j}\right] \text { modulo } \Delta^{\perp}
$$

which implies that $\left[\bar{\tau}_{i}, \bar{\tau}_{j}\right] \in \Delta^{\perp}$.

Sufficiency: Assume that for one choice of $\bar{\tau}_{1}$ satisfying (10), we have $\left[\bar{\tau}_{i}, \bar{\tau}_{j}\right] \in \Delta^{\perp}$ for $1 \leq, i, j \leq r$ where $\tau_{i}$ was defined in (11). Then we need to prove that there exist $H_{i} \in \Delta^{\perp}$ for $1 \leq i \leq r$ such that $\left[\tau_{i}, \tau_{j}\right]=0$ where $\tau_{k}=\bar{\tau}_{k}+H_{k}$.

Let $\left\{\tau_{r+1}, \cdots, \tau_{n}\right\}$ be $(n-r)$ determined commutative basis of $\Delta^{\perp}$, it is well-known (see Nijmeijer and Van der Schaft (1990)) that there exist $r$ commutative vector fields, noted as $\left\{X_{1}, \cdots, X_{r}\right\}$, such that they commute with $\left\{\tau_{r+1}, \cdots, \tau_{n}\right\}$, thus form together a basis of the tangent fiber bundle $T \mathscr{X}$ of $\mathscr{X}$.

Then, for all $1 \leq i \leq r$ we can rewrite $\bar{\tau}_{i}$ as:

$$
\bar{\tau}_{i}=\sum_{l=1}^{r} \gamma_{i, l}(x) X_{l}+\sum_{l=r+1}^{n} \gamma_{i, l}(x) \tau_{l}
$$

By choosing $H_{i}=-\sum_{l=r+1}^{n} \gamma_{i, l}(x) \tau_{l} \in \Delta^{\perp}$, then we have:

$$
\tau_{i}=\bar{\tau}_{i}+H_{i}=\sum_{l=1}^{r} \gamma_{i, l}(x) X_{l}
$$

For the same reason, there exist $H_{j}=\sum_{l=r+1}^{n} \gamma_{j, l}(x) \tau_{l} \in \Delta^{\perp}$ yielding:

$$
\tau_{j}=\bar{\tau}_{j}+H_{j}=\sum_{l=1}^{r} \gamma_{j, l}(x) X_{l}
$$

Moreover, for $1 \leq i, j \leq r$, due to Lemma 3 we have

$$
\left[\bar{\tau}_{i}, \bar{\tau}_{j}\right]=\left[\tau_{i}, \tau_{j}\right] \text { modulo } \Delta^{\perp}
$$

As $\left[X_{k}, X_{s}\right]=0$ for $1 \leq k, s \leq r$, then (33) becomes

$$
\left[\bar{\tau}_{i}, \bar{\tau}_{j}\right]=\left[\sum_{l, s=1}^{r} \gamma_{i, l} L_{X_{l}} \gamma_{j, s} X_{s}-\sum_{s, l=1}^{r} \gamma_{j, s} L_{X_{s}} \gamma_{i, l} X_{l}\right] \text { modulo } \Delta^{\perp}
$$

Thus, if $\left[\bar{\tau}_{i}, \bar{\tau}_{j}\right] \in \Delta^{\perp}$, then there exist $H_{i} \in \Delta^{\perp}$ and $H_{j} \in \Delta^{\perp}$ defined above, such that

$$
\left[\tau_{i}, \tau_{j}\right]=\sum_{l, s=1}^{r} \gamma_{i, l} L_{X_{l}} \gamma_{j, s} X_{s}-\sum_{s, l=1}^{r} \gamma_{j, s} L_{X_{s}} \gamma_{i, l} X_{l}=0
$$

due to the fact that $X_{k} \notin \Delta^{\perp}$.

\section{Acknowledgement}


The authors gratefully acknowledge anonymous reviewers for the valuable suggestions and helpful remarks to improve the quality our manuscript.

\section{References}

Back, J., Yu, K. T., Seo, J. H., 2006. Dynamic observer error linearization. Automatica 42 (12), 2195-2200.

Besançon, G., 2007. Nonlinear Observers and Applications. Lecture notes in control and information sciences. Springer.

Bestle, D., Zeitz, M., 1983. Canonical form observer design for nonlinear time varying systems. International Journal of Control 38, 419-431.

Boutat, D., 2015. Extended nonlinear observer normal forms for a class of nonlinear dynamical systems. International Journal of Robust and Nonlinear Control 25 (3), 461-474.

Boutat, D., Benali, A., Hammouri, H., Busawon, K., 2009. New algorithm for observer error linearization with a diffeomorphism on the outputs. Automatica 45 (10), 2187-2193.

Boutat, D., Busawon, K., 2011. On the transformation of nonlinear dynamical systems into the extended nonlinear observable canonical form. International Journal of Control 84 (1), 94-106.

Boutat, D., Zheng, G., Barbot, J. P., Hammouri, H., 2006. Observer error linearization multi-output depending. In: 45th IEEE Conference on Decision and Control.

Feng, R., Garrido, J., 2008. Actuarial applications of epidemiological models. Concordia University. Department of Mathematics \& Statistics.

Guay, M., 2002. Observer linearization by output-dependent time-scale transformations. IEEE Transactions on Automatic Control 47 (10), 1730-1735.

Isidori, A., 1995. Nonlinear Control Systems. Communications and Control Engineering. Springer.

Jo, H. N., Seo, H. J., 2002. Observer design for non-linear systems that are not uniformly observable. International Journal of Control 75 (5), 369-380.

Jouan, P., 2003. Immersion of nonlinear systems into linear systems modulo output injection. SIAM Journal on Control and Optimisation 41 (6), 17561778 .

Kang, W., Barbot, J., Xu, L., 2009. On the observability of nonlinear and switched systems. Emergent Problems in Nonlinear Systems and Control, 199-216.

Keller, H., 1987. Nonlinear observer design by transformation into a generalized observer canonical form. International Journal of Control 46 (6), 19151930.

Krener, A., Isidori, A., 1983. Linearization by output injection and nonlinear observers. Systems \& Control Letters 3 (1), 47-52.

Krener, A., Respondek, W., 1985. Nonlinear observers with linearizable error dynamics. SIAM Journal on Control and Optimization, 23 (2), 197-216.

Lynch, A., Bortoff, S., jun 2001. Nonlinear observers with approximately linear error dynamics: the multivariable case. IEEE Transactions on Automatic Control 46 (6), 927-932.

Marino, R., Tomei, P., 1996. Nonlinear control design: geometric, adaptive and robust.

Nijmeijer, H., Van der Schaft, A., 1990. Nonlinear dynamical control systems. Springer.

Noh, D., Jo, N., Seo, J., 2004. Nonlinear observer design by dynamic observer error linearization. IEEE Transactions on Automatic Control 49 (10), 17461753.

Phelps, A., 1991. On constructing nonlinear observers. SIAM Journal on Control and Optimmisation 29, 516-534.

Raggett, G., 1982. Modeling the eyam plague. The Institute of Mathematics and its Applications Journal 18, 221-226.

Respondek, W., Pogromsky, A., Nijmeijer, H., 2004. Time scaling for observer design with linearizable error dynamics. Automatica, 40 (2), 277-285.

Robenack, K., Lynch, A., 2006. Observer design using a partial nonlinear observer canonical form. International Journal of Applied Mathematics and Computer Science 16 (3), 333-343.

Tami, R., Boutat, D., Zheng, G., 2013. Extended output depending normal form. Automatica 49 (7), 2192-2198.

Tami, R., Boutat, D., Zheng, G., 2014a. Nonlinear observer for the pm synchronous motor. In: American Control Conference (ACC), 2014.

Tami, R., Boutat, D., Zheng, G., Kratz, F., 2014b. Parameters and states estimation for dengue epidemic model. In: 13th European Control Conference.
Trinh, H., Fernando, T., Nahavandi, S., 2006. Partial-state observers for nonlinear systems. IEEE Transactions on Automatic Control 51 (11), 1808-1812.

Xia, X., Gao, W., 1989. Nonlinear observer with linearizable error dynamics. SIAM Journal on Control and Optimmisation 27, 199-216.

Zheng, G., Boutat, D., 2011. Synchronisation of chaotic systems via reduced observers. IET Control Theory \& Applications 5 (2), 308-314.

Zheng, G., Boutat, D., Barbot, J., 2007. A single output dependent observability normal form. SIAM Journal on Control and Optimization 46 (6), 2242 2255 .

Zheng, G., Boutat, D., Barbot, J.-P., 2005. Output dependent observability linear normal form. In: 44th IEEE Conference on Decision and Control.

Zheng, G., Boutat, D., Barbot, J.-P., 2009. Multi-output dependent observability normal form. Journal of Nonlinear Analysis Series A: Theory, Methods \& Applications 70 (1), 404-418. 